\title{
Etats limites plastiques en présence de l'écrouissage isotrope
}

\author{
Ali Chaaba* — Lahbib Bousshine** \\ * Ecole Nationale Supérieure d'Arts et Métiers \\ Marjane II, Beni M'hamed, B.P. 4024, Meknès Maroc \\ a.chaaba@yahoo.com \\ ** Ecole Nationale Supérieure d'Electricité et de Mécanique \\ B.P. 8118, Oasis, Casablanca, Maroc
}

\begin{abstract}
RÉSUMÉ. Que ce soit dans un objectif de conception de structures ou de mise en forme par déformation plastique, l'analyse limite est parmi les méthodes directes d'estimation des états limites plastiques les plus connues. En se basant sur l'approche cinématique au sens de l'analyse limite et en se servant de la méthode des éléments finis, l'objectif principal de ce travail consiste à aborder les grandes déformations plastiques du modèle de von Mises en considérant l'écrouissage isotrope. Afin de surmonter la difficulté engendrée par la nondifférentiabilité de la dissipation plastique, nous avons particularisé une méthode de régularisation initialement développée pour les matériaux compressibles. Dans le but de suivre les séquences de grandes déformations, la notion d'analyse limite séquentielle qui consiste en la mise à jour des propriétés des matériaux et de la géométrie de configuration après chaque séquence est appliquée.
\end{abstract}

ABSTRACT: Either in Structural Engineering or Metalworking processes by plastic deformation, the limit analysis is among the direct methods of the plastic collapse assessment. Basing on the kinematical approach in the sense of limit analysis and using finite elements method, the main objective of this work is to deal with large plastic deformations of von Mises's materials taking into account isotropic hardening. In order to overcome difficulties caused by the non differentiability of plastic dissipation, we adopt a regularization method originally developed for compressible materials. In order to follow up the sequences of large deformations, we have used the sequential limit analysis procedure; it consists in the updating of material properties and geometrical configuration after each sequence.

MOTS-CLÉS : analyse limite, écrouissage, programmation mathématique, méthode des éléments finis.

KEYWORDS: limit analysis, strain hardening, mathematical programming, finite element method.

DOI:10.3166/EJCM.18.525-545 (C) 2009 Lavoisier, Paris 


\section{Introduction}

En plasticité, les méthodes élastoplastiques incrémentales déduites des équations générales de la mécanique des solides déformables et des lois de la plasticité, permettent l'obtention des contraintes et des déformations sur une histoire de chargement jusqu'à l'atteinte du mécanisme d'écoulement plastique (Koiter, 1960 ; Halphen et Salençon, 1987 ; Fassi Fihri et al., 2003, 2004 ; et autres). Cet état limite d'écoulement plastique pourra être obtenu par des méthodes directes, adoptant le comportement rigide-plastique (Kim et Yang, 1985 ; Kobayashi et al., 1989; Lee et Kobayashi, 1973 ; Lung et Marenholtz, 1974 ; Mori et al., 1982 ; Nagtegaal et De Jong, 1981 ; Yoon et Yang, 1988, Wagoner et Chenot , 2001) ou par l'adoption des méthodes d'analyse limite, sans effectuer les calculs intermédiaires élastoplastiques qui sont nécessaires lors de l'adoption des méthodes incrémentales (pas à pas). Les approches d'analyse limite (Prager, 1952 ; Koiter, 1958 ; Salençon, 1983 et autres), qui adoptent généralement le comportement rigide parfaitement plastique du matériau, se basent essentiellement sur les principes variationnels tels que celui de Markov comme une formulation cinématique et celui de Hill comme une formulation statique. Les procédures d'analyse limite ont trouvé une large application dans les domaines de l'ingénieur tels que la mise en forme des métaux, le dimensionnement optimal aux états limites plastiques, et en géotechnique... A titre indicatif, notons qu'il existe principalement, outre l'analyse limite, deux autres méthodes qui permettent la détermination de manière directe de l'état limite plastique du solide rigide parfaitement plastique, la méthode des tranches et la méthode des lignes de glissement (Hill, 1950 ; Johnson et al., 1982 et autres). Ces deux méthodes qui ont le mérite d'être plus ou moins analytiques ou semi-analytiques se heurtent à des difficultés majeures. En fait, elles deviendraient très lourdes et difficiles à appliquer pour des cas avec des géométries ou des conditions aux limites plus ou moins complexes. Il convient de noter que ces deux méthodes ne sont que des exemples d'application en 2D avec des choix particuliers de champs (contraintes ou vitesses de déformations) de l'analyse limite (Salençon, 1983).

La procédure d'analyse limite, qui a gagné en popularité ces dernières années, est basée sur deux théorèmes fondamentaux (Hill, 1951, 1952; Greenberg et Prager, 1951 ; Drucker et al., 1951, 1952) qui sont le théorème de la borne inférieure ou théorème statique et le théorème de la borne supérieure ou théorème cinématique, à travers lesquels la méthode est appliquée effectivement aux problèmes pratiques (on cite par exemple: Save et Massonet, 1972; Chen, 1975). Ces théorèmes fournissent les bornes inférieure et supérieure de la charge limite "exacte». Néanmoins, vu sa simplicité de mise en œuvre, la méthode cinématique est plus utilisée surtout au niveau numérique. La version primitive de cette approche, qui est appliquée surtout pour les solides $2 \mathrm{D}$, consiste à proposer un champ de vitesse tel que le solide déformé plastiquement est supposé divisé en plusieurs blocs, généralement triangulaires, qui restent rigides mais séparés de leurs voisins par des surfaces de discontinuité de vitesse tangentielle (par exemples : Avitzur, 1968, 1983, Chen, 1975). Le champ de vitesse doit être compatible avec les conditions 
extérieures imposées. Le point faible de cette approche analytique réside également dans le fait que la proposition de l'architecture d'un mécanisme de ruine plastique est généralement délicate surtout pour des problèmes ayant une géométrie ou des conditions aux limites complexes.

Cette carence a ouvert la voie au développement des algorithmes numériques qui peuvent fournir simultanément le champ de vitesse et la charge limite. La méthode cinématique de l'analyse limite qui se traduit par la résolution d'un problème de programmation mathématique dont la fonction objectif contenant la dissipation plastique est non linéaire et surtout non différentiable, ce qui a rendu son traitement numérique plus délicat. La non-différentiabilité de la fonction objectif est une source de difficultés pour les codes d'optimisation utilisant les algorithmes classiques de la programmation mathématique qui exigent la connaissance des gradients des fonctions à minimiser. Pour surmonter cette difficulté, plusieurs auteurs (Friaâ, 1978 ; Guennouni et Letallec, 1982 ; Guennouni, 1982 ; Gaudrat, 1991 ; Voldoire, 2000, 2003 ) ont proposé et adopté des méthodes de régularisation. D’autres auteurs (par exemple Ponter et al., 1997, 2000) ont exploité une succession de calculs élastiques linéaires pour approcher la solution rigide-plastique.

Dans ce travail, nous avons adopté une méthode de régularisation qui consiste à ajouter aux vitesses de déformation plastique une partie visqueuse linéaire fictive de telle sorte que l'énergie totale tend vers la dissipation plastique quand les paramètres de la viscosité deviennent suffisamment grands. Cette procédure de régularisation, initialement développée pour l'analyse limite du matériau de Drucker-Prager comme un matériau compressible (Chaaba, 2001; Chaaba et al., 2003), découle d'une méthode plus générale qui est basée sur le produit d'inf convolution, dû initialement à Moreau (1965). L'importance de cette approche que nous avons adoptée réside dans le fait qu'elle permet de rendre différentiable une fonction sous différentiable (cas de la dissipation) en effectuant son produit d'inf-convolution avec une autre fonction auxiliaire différentiable. En outre, cette façon de procéder fournit un moyen simple approximatif de calcul du champ de contrainte comme étant le gradient de la fonction résultant de l'inf-convolution.

L'objet de ce travail est de mettre au point une approche algorithmique de calcul des états limites plastiques non seulement pour un solide rigide parfaitement plastique mais aussi pour un solide ayant un comportement plastique écrouissable isotrope, obéissant au principe de travail maximum de Hill (1950). Rappelons qu'historiquement, les résultats de l'analyse limite ne sont applicables qu'aux solides déformables, sous l'hypothèse de petites déformations, rigides parfaitement plastiques éliminant ainsi toute notion d'écrouissage, propriété généralement présentée par le comportement plastique des métaux. En adoptant la notion d'analyse limite séquentielle, initialement proposée par Horne (1965) et utilisée ensuite par d'autres auteurs (par exemples : Yang, 1993 ; Huh et al., 1999 ; 2001 ; Corradi et Panzeri, 2004 ; Leu, 2007, ainsi que d'autres), on a pu répondre à la question de l'écrouissage et au problème de grandes déformations en résolvant une séquence de problèmes d'analyse limite. En effet, par actualisation de la géométrie après chaque 
séquence et de la contrainte limite d'écoulement plastique, nous pouvons suivre la déformation de la pièce jusqu'à un niveau avancé. Un problème de compression d'une pièce métallique, obéissant au critère de von Mises avec ou sans écrouissage, en petites ou en grandes déformations, fera l'objet d'une application des algorithmes qui seront présentés dans cet article.

\section{Ecoulement plastique de von Mises et écrouissage isotrope}

Le milieu continu à déformer est supposé homogène, isotrope, incompressible, isotherme et obéissant au modèle de von Mises dont le critère est exprimé par l'équation $f(\sigma)=\sigma_{e q}-\sigma_{Y}=0$, où $\sigma_{e q}$ est la contrainte équivalente de von Mises (Lemaître et Chaboche, 1990) et $\sigma_{Y}$ est la contrainte limite d'écoulement plastique, propriété propre au matériau en déformation. Notons par $k$ la contrainte limite de cisaillement exprimée pour le critère de von Mises par $k=\sigma_{Y} / \sqrt{3}$. La loi d'écoulement plastique est précisée par la loi de normalité classique $\dot{\varepsilon}^{p}=\dot{\lambda} \frac{\partial f}{\partial \sigma}$ et la densité de dissipation plastique est exprimée par $D\left(\dot{\varepsilon}^{p}\right)=k\left\|\dot{e}^{p}\right\|$, où $\left\|\dot{e}^{p}\right\|$ est la norme euclidienne du déviateur du tenseur des vitesses de déformation plastique, exprimée par : $\left\|\dot{e}^{p}\right\|=\sqrt{\operatorname{Trace}\left(\dot{e}^{p}\right)^{2}}$. Pour un matériau ayant un comportement rigide parfaitement plastique, la contrainte limite d'écoulement est constante. Cependant, l'écrouissage est généralement un phénomène qui se manifeste lors du processus de déformation plastique des métaux. Ainsi pour caractériser ce phénomène en supposant qu'il est de type isotrope, la contrainte limite d'écoulement plastique est considérée non plus constante mais elle sera une fonction de la déformation plastique effective accumulée; cette dernière étant définie par l'expression (Lemaître et Chaboche, 1990) :

$$
\varepsilon_{p}=\int \sqrt{2 / 3}\left(\dot{\varepsilon}_{i j}^{p} \cdot \dot{\varepsilon}_{i j}^{p}\right)^{1 / 2} d t
$$

Notons cette fonction d'écrouissage par $\beta\left(\varepsilon_{p}\right)$ traduisant l'aspect évolutif de la contrainte limite actuelle, notée $\sigma_{Y}^{a}$, en fonction de la déformation plastique accumulée $\varepsilon_{p}$; Cette loi est généralement écrite sous l'une des deux formes en puissance (Hwan, 1992 ; Huh et al., 1999) :

$$
\beta\left(\varepsilon_{p}\right)=\frac{\sigma_{Y}^{a}}{\sigma_{Y}}=1+M \varepsilon_{p}^{n} \text { ou } \beta\left(\varepsilon_{p}\right)=\frac{\sigma_{Y}^{a}}{\sigma_{Y}}=\left(1+M \varepsilon_{p}\right)^{n}
$$

$M$ et $n$ sont des paramètres matériels supposés constants caractérisant la loi d'écrouissage. Dans ce cas d'écrouissage, le potentiel de dissipation $D\left(\dot{\varepsilon}^{p}\right)$ a pour expression : 


$$
D\left(\dot{\varepsilon}^{p}\right)=k \beta\left(\varepsilon_{p}\right)\left\|\dot{\varepsilon}^{p}\right\|
$$

Nous précisons que la déformation effective $\varepsilon_{p}$, présente dans les expressions $[1,2$ et 3 ], ne sera considérée que comme un simple paramètre et calculée après chaque séquence et sa valeur sera exploitée lors de la détermination de la séquence suivante.

\section{Formulation cinématique de l'analyse limite}

Le cadre général de l'analyse limite des métaux considère le comportement rigide parfaitement plastique du matériau constitutif du solide déformable en question (la déformation élastique étant négligée). Le milieu occupe un volume $\Omega$ de frontière $S$, soumis à des forces de traction de surface $\bar{t}$ non nulles sur la partie $S_{1}$ de $S$. Vu la grandeur des forces nécessaires au processus de déformation plastique des métaux, les forces de volume sont considérées comme négligeables. Les conditions aux limites cinématiques sont imposées en termes de vitesses nulles sur la partie $S_{0}$ de $S$. En analyse limite, le chargement extérieur est choisi proportionnel à un chargement de référence $\bar{t}^{0}$ tel que : $\bar{t}=\alpha \bar{t}^{0}$ où $\alpha$ est un nombre réel non négatif, appelé facteur de charge.

Soit $\dot{u}^{c}$ un champ de vitesse qui est cinématiquement admissible (C.A) et plastiquement admissible (P.A) tel que :

$$
\begin{aligned}
& \dot{\varepsilon}\left(\dot{u}^{c}\right)=\operatorname{grad}_{s} \dot{u}^{c} \text { dans } \Omega, \text { et } \dot{u}^{c}=0 \text { sur } S_{0} \\
& \int_{S_{1}} \dot{u}^{c} \cdot \bar{t}^{0} d S>0
\end{aligned}
$$

En utilisant le principe des puissances virtuelles, le facteur de charge cinématique $\alpha^{c}$ correspondant au champ de vitesse $\dot{u}^{c}$ décrivant un mécanisme de ruine admissible est défini par l'expression de l'égalité de la puissance totale par déformation plastique et la puissance du chargement extérieur (Koiter, 1960 ; Chaaba et al., 2003) :

$$
\int_{\Omega} D\left(\dot{\varepsilon}\left(\dot{u}^{c}\right)\right) d \Omega=\alpha^{c} \int_{S_{1}} \dot{u}^{c} \cdot \bar{t}^{0} d S
$$

En considérant l'expression [3], de la condition [4b] et l'égalité [5], le facteur de charge cinématique $\alpha^{c}$, associé à un champ de vitesse admissible $\dot{u}^{c}$, peut s'écrire sous la forme suivante :

$$
\alpha^{c}=\frac{k \beta\left(\varepsilon_{p}\right) \int_{\Omega}\|\dot{\varepsilon}\| d \Omega}{\int_{S_{1}} \dot{u}^{c} \cdot \bar{t}^{0} d S}
$$

La théorie d'analyse limite n'est rendue applicable aux problèmes pratiques qu'après avoir énoncé les deux théorèmes extrémaux de la borne inférieure 
(théorème statique) et de la borne supérieure (théorème cinématique). Nous sommes restreints ici à rappeler l'énoncé du théorème cinématique de la borne supérieure (par exemple: Koiter, 1960; Salençon, 2002 et autres) sur lequel nous nous baserons par la suite.

Théorème de la borne supérieure: Soit $\alpha^{l}$ le multiplicateur limite correspondant au mécanisme de ruine décrit par le champ de vitesse $\dot{u}$ de l'état limite, et $\alpha^{c}$ un multiplicateur cinématique associé au champ de vitesse admissible $\dot{u}^{c}$. Alors on $a$ :

$$
\forall \dot{u}^{c} \text { admissible, } \alpha^{c} \geq \alpha^{l} .
$$

En se basant sur l'égalité [6] laquelle définit le facteur de charge cinématique $\alpha^{c}$, la condition de normalisation $\int_{S_{1}} \dot{u}^{c} \cdot \bar{t}^{0} d S=1$, et le théorème précédent de la borne supérieure, la recherche de l'état limite plastique se traduit par la résolution du problème de programmation mathématique non linéaire suivant :

$$
\begin{aligned}
& \text { Inf } k \beta\left(\varepsilon_{p}\right) \int_{\Omega}\|\dot{\varepsilon}\| d \Omega \\
& \dot{u}^{c} \text { admissible, } \dot{e}_{m}=0 \text { dans } \Omega \text { et } \int_{S_{1}} \dot{u}^{c} \cdot \bar{t}^{0} d S=1
\end{aligned}
$$

où $\dot{e}_{m}$ est la trace du tenseur de vitesse de déformation plastique. Le problème d'analyse limite est ainsi un problème d'optimisation non linéaire avec contraintes imposant les forces de surface. En général, $\bar{t}^{0}$ est une pression que nous supposons repartie de manière uniforme sur la partie $S_{1}$ de la frontière. Cette façon de faire est celle présentée dans (Chaaba, 2001 ; Bousshine et al., 2002 ; Chaaba et al., 2003). Autrement, au lieu de spécifier les forces sur la partie $S_{1}$ de la frontière de la pièce à déformer, on impose la vitesse sur la même zone $S_{1}$ (Hwan, 1992 ; Huh et al., 1999 ; Leu, 2007 , 2008). C'est le cas par exemple du mouvement de translation de la matrice supérieure d'une presse de mise en forme. L'intérêt de cette remarque permet ainsi d'éliminer la condition de normalisation dans le programme d'optimisation :

$$
\begin{gathered}
\text { Inf } k \beta\left(\varepsilon_{p}\right) \int_{\Omega}\|\dot{\varepsilon}\| d \Omega \\
\begin{cases}\dot{e}_{m}=0 & \text { dans } \Omega \\
\dot{u}^{c}=0 & \text { sur } S_{0} \\
\dot{u}^{c} \neq 0 & \text { sur } S_{1}\end{cases}
\end{gathered}
$$

Insistons sur la fait que la dissipation $D(\dot{\varepsilon})$ est une fonction convexe mais n'est pas différentiable par rapport aux variables $\dot{u}^{c}$ dans les régions restant rigides lors de la déformation de la structure. Afin de surmonter la difficulté numérique causée par le caractère non différentiable de cette fonction, nous proposons de régulariser la 
fonction objectif au moyen de l'inf-convolution dont le principe est résumé et présenté dans (Chaaba, 2001 ; Chaaba et al., 2003).

\section{Aspect algorithmique de l'approche cinématique}

La dissipation plastique est seulement sous-différentiable et le champ de contraintes ne pourra être déduit jusqu'ici qu'au moyen d'une inclusion sousdifférentielle (Rockafellar, 1996). La régularisation a donc pour objectif principal d'approcher le gradient de la fonction objectif, toujours exigé par les algorithmes déterministes de la programmation mathématique et de calculer approximativement le champ des contraintes.

On note par $d(\dot{\varepsilon})$ la fonction obtenue par inf-convolution de la fonction auxiliaire et la dissipation plastique $D\left(\dot{\varepsilon}^{p}\right)$ par rapport aux vitesses de déformation plastique $\dot{\varepsilon}^{p}$. En faisant référence à (Chaaba, 2001; Chaaba et al., 2003), le problème d'analyse limite sous sa forme régularisée est :

$$
\begin{aligned}
& \text { Inf } \int_{\Omega} d\left(\dot{\varepsilon}\left(\dot{u}^{c}\right)\right) d \Omega \\
& \dot{u}^{c}=0 \text { sur } S_{0} \\
& \dot{u}^{c} \neq 0 \text { sur } S_{1}
\end{aligned}
$$

Il convient de noter que d'autres auteurs (par exemples : Mercier, 1977 ; Friaâ, 1979 ; Gaudrat, 1991) ont utilisé d'autres approches et abouti au même type de formulation [10]. Sur la base de cette transformation, le champ de contrainte est approché localement par le gradient de la fonction dissipation régularisée : $\sigma=\frac{\partial d(\dot{\varepsilon})}{\partial \dot{\varepsilon}}$. La fonction $d(\dot{\varepsilon})$, dans le cas du modèle de von Mises prendra la forme suivante :

$$
\begin{aligned}
& \text { si }\|\dot{e}\| \geq \frac{k \beta\left(\varepsilon_{p}\right)}{2 \mu^{*}}, \text { alors }\left\|\dot{e}^{p}\right\|=\left(\|\dot{e}\|-\frac{k \beta\left(\varepsilon_{p}\right)}{2 \mu^{*}}\right) \\
& \text { et } d(\dot{\varepsilon})=\frac{K_{c}^{*}}{2}\left(\dot{e}_{m}\right)^{2}+\mu^{*}\left\{\|\dot{e}\|^{2}-\left(\|\dot{e}\|-\frac{k \beta\left(\varepsilon_{p}\right)}{2 \mu^{*}}\right)^{2}\right\} \\
& \text { sinon, } \quad\left\|\dot{e}^{p}\right\|=0 \text { et } \quad d(\dot{\varepsilon})=\frac{K_{c}^{*}}{2}\left(\dot{e}_{m}\right)^{2}+\mu^{*}\|\dot{e}\|^{2}
\end{aligned}
$$

où $\dot{e}_{m}$ est la trace du tenseur vitesse de déformation $\dot{\varepsilon}: \dot{e}_{m}=\operatorname{trace}(\dot{\varepsilon})$. Dans l'expression [11], on voit apparaître la fonction d'écrouissage $\beta\left(\varepsilon_{p}\right)$ traduisant donc à chaque séquence d'analyse limite l'effet de ce phénomène. Les grandeurs $K_{c}^{*}$ et $\mu^{*}$ sont les paramètres de la régularisation définis en fonction d'un module de 
Young et d'un coefficient de Poisson fictifs tels que: $K_{c}^{*}=\frac{E^{*}}{3\left(1-2 v^{*}\right)}$ et $\mu^{*}=\frac{E^{*}}{2\left(1+v^{*}\right)}$. Leurs valeurs numériques moyennes à adopter pour les applications numériques seront précisées ultérieurement. Par ailleurs, nous signalons que l'algorithme présenté ci-avant par $[11,12]$ coïncide avec celui présenté dans Jospin (1992) appliqué au calcul des états limites des coudes rigides parfaitement plastiques. Notre application concerne surtout les problèmes d'états plans de déformation, états axisymétriques ou des solides 3D avec ou sans écrouissage, en petites ou en grandes déformations.

Discrétisation en éléments finis. Quand l'approche cinématique est adoptée afin de déterminer l'état limite plastique, on est conduit automatiquement au choix d'une approximation cinématiquement admissible du champ de vitesses de déplacement. Le champ de vitesse de déformation s'en déduit facilement par les relations déformations-vitesse de déplacement. Ainsi, la dissipation totale deviendrait comme une fonction seulement des vitesses aux nœuds. Soit $N(x)$ la matrice des fonctions de forme, $B(x)$ la matrice contenant les dérivées premières des fonctions de forme et $\dot{U}$ le vecteur des vitesses nodales, nous rappelons les formules usuelles suivantes:

$$
\dot{u}(x)=N(x) \dot{U} ; \dot{\varepsilon}(x)=B(x) \dot{U}
$$

Dans cette formulation, le champ de contrainte n'est pas discrétisé. Sa valeur est déterminée, en chaque point d'intégration de Gauss, par les relations de comportement. Ainsi, le problème de la borne supérieure s'écrit sous sa forme discrétisée suivante :

$$
\begin{aligned}
& \text { Inf } \int_{\Omega} d(B(x) \dot{U}) d \Omega \\
& \text { Conditions aux limites sur } S_{0} \text { et } S_{1}
\end{aligned}
$$

accompagné avec les équations de contraintes en chaque point de Gauss: $\sigma=\frac{\partial d(B(x) \dot{U})}{\partial \dot{\varepsilon}}$. Soit $s$ le déviateur des contraintes et $s_{m}$ la pression hydrostatique, définis par $s_{m}=\operatorname{Trace}(\sigma) / 3$ et $s=\sigma-s_{m} I$, où $I$ est la matrice unité d'ordre 3 . En tenant compte de l'algorithme de la régularisation adoptée [11-12], le gradient de la fonction objectif dans [14] est donné par $\sigma=s_{m} I+s$ tel que :

$$
s_{m}=K_{c}^{*}\left(\dot{e}_{m}-\dot{e}_{m}^{p}\right) \quad \text { et } \quad s=2 \mu^{*}\left(\dot{e}-\dot{e}^{p}\right)
$$

L'algorithme de résolution pourra être illustré par l'organigramme simplifié, présenté à la fin de cette section. Nous notons que la résolution du problème d'optimisation [14] est réalisée à l'aide du logiciel Minos (Murtagh et Saunders, 1987). Nous avons inséré dans l'organigramme la façon avec laquelle la géométrie et le seuil de plasticité sont actualisés. En exploitant le vecteur de vitesses aux nœuds qu'on a obtenu dans la séquence présente d'analyse limite [14], la séquence suivante 
d'analyse limite est conduite en résolvant à nouveau l'équation [14] mais après actualisation de la configuration géométrique en multipliant le champ de vitesse par un incrément de temps $\Delta t$ suffisamment petit (validité de l'hypothèse de petites perturbations) telle que $x=x+\dot{U} \Delta t$ et $y=y+\dot{U} \Delta t$ (pour un problème 2D). Tandis que le seuil de plasticité $\sigma_{Y}$ est actualisé à travers le calcul tout d'abord de la vitesse de déformation plastique accumulée $\dot{\varepsilon}_{p}$ et ensuite par multiplication de cette quantité par $\Delta t$ telle que $\varepsilon_{p}=\varepsilon_{p}+\dot{\varepsilon}_{p} \Delta t$. Une des deux équations [2] est adoptée alors pour mettre à jour $\sigma_{Y}$ conduisant donc à tenir compte de l'écrouissage isotrope.

REMARQUE. - Dans le but d'obtenir un problème d'optimisation mieux conditionné, une mise à l'échelle du vecteur des vitesses nodales $\dot{U}$ et de la fonction objective $F O(\dot{U})$ est adoptée comme suit :

$$
\overline{\dot{U}}=\dot{U} / \dot{U}_{R} \text { et } \bar{F}=F O(\dot{U}) / d_{R},
$$

où $\dot{U}_{R}$ est un déplacement de référence et $d_{R}=E_{R}^{*} \dot{U}_{R} \dot{U}_{R} / 2$ dans laquelle $E_{R}^{*}$ est un module de Young fictif de référence.

Dans la suite, notre objectif consiste à répondre à deux questions. La première concerne l'estimation des valeurs numériques des paramètres fictifs de l'algorithme de régularisation qui sont le module de Young $E^{*}$ et le coefficient de Poisson $v^{*}$. La seconde question traite de l'influence de l'effet de l'écrouissage isotrope sur la charge limite et/ou de l'effet de l'actualisation de la géométrie lors du processus de déformation.

\section{Organigramme}

\section{Notation}

- FO : Dissipation totale (fonction objectif du problème d'optimisation [14])

- $G$ : Vecteur gradient de la fonction objectif $F O$. (Ces deux grandeurs $F O$ et $G$ sont initialisées à zéro)

$-\varepsilon_{t o l}:$ Tolérance de précision concernant le processus de convergence de l'algorithme.

- $\Delta t$ : Incrément de temps qui doit être choisi suffisamment petit pour garantir l'hypothèse des petites déformations)

- $\Delta x$ et $\Delta y$ (problème 2D) représentent les incréments des coordonnées du déplacement aux nœuds du maillage, obtenus en multipliant le champ de vitesse nodales $\dot{U}$ par l'incrément de temps $\Delta t$.

$-\sigma^{i+1}$ et $\sigma^{i}$ sont les valeurs des contraintes (aux points de Gauss) respectivement dans l'itération $\mathrm{i}+1$ et l'itération $\mathrm{i}$. 


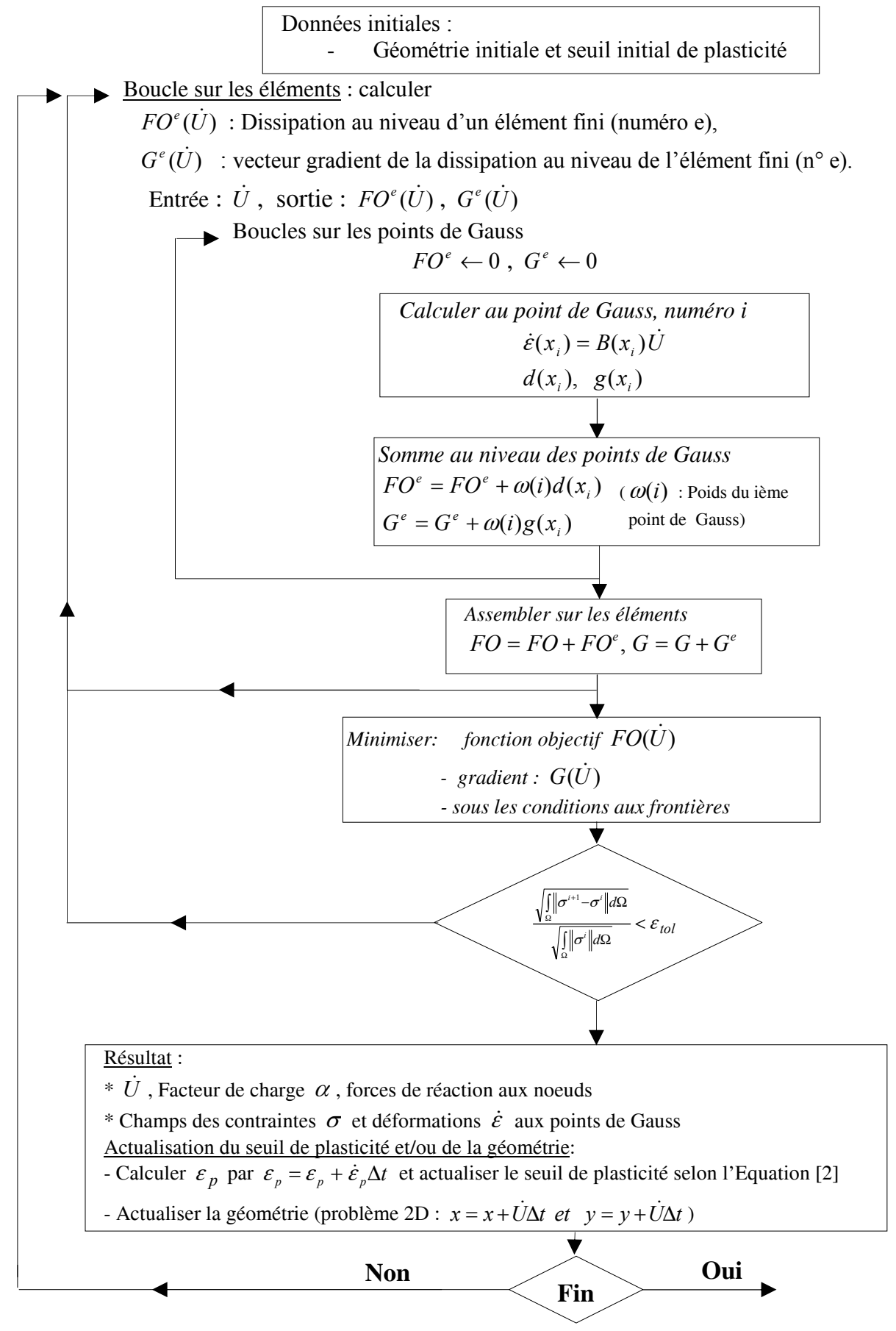




\section{Ordres de grandeur des paramètres fictifs de l'algorithme de régularisation}

Notre objectif principal dans cette section consiste à estimer les bonnes valeurs du module de Young et du coefficient de Poisson fictifs. Nous avons choisi de réaliser cette étude sur une application analytique simple d'une pièce métallique carrée en traction ou compression et sur un exemple numérique relativement difficile qui est le poinçonnement d'un milieu semi-infini. Dans cette section, on n'est pas intéressé ni par l'aspect écrouissage ni par l'actualisation de la géométrie.

\subsection{Coefficient de Poisson : un exemple analytique}

Cet exemple simple considère une structure, en état plan de déformation, de forme carrée dans le plan $(x, y)$ constituée d'un matériau rigide parfaitement plastique obéissant au critère de von Mises de contrainte limite de cisaillement $k$ $\left(k=\sigma_{Y} / \sqrt{3}=30 \mathrm{MPa}\right)$, sollicitée en compression uniaxiale selon la direction y. Il est supposé que la structure bidimensionnelle est en état de déformation homogène. Soit $\dot{u}_{x}$ et $\dot{u}_{y}$ respectivement les vitesses horizontale et verticale des deux côtés ( $\dot{u}_{z}=0$, d'après l'hypothèse d'état plan de déformation). La dissipation plastique en fonction du coefficient de Poisson fictif est présentée sur la figure 1. En tenant compte de la condition de normalisation, $D(\dot{\varepsilon})$ est la dissipation de von Mises et elle est fonction dans ce cas, seulement de la vitesse $\dot{u}_{x}$ :

$D(\dot{\varepsilon})=k\|\dot{e}(\dot{\varepsilon})\|=k \sqrt{2 / 3}\left(\dot{u}_{x}^{2}+\dot{u}_{x}+1\right)^{1 / 2}$.

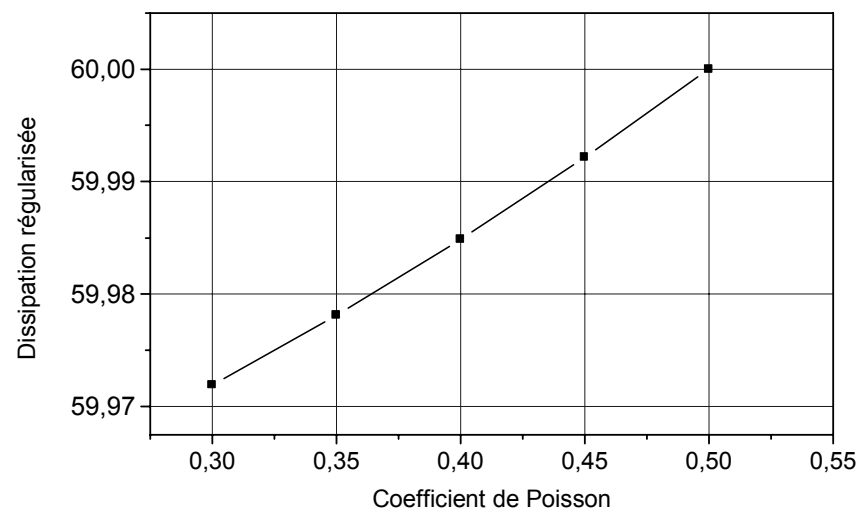

Figure 1. Effet du coefficient de Poisson, $\left(E=10^{5}\right)$ 
Si on tient compte directement de la condition d'incompressibilité fournissant $\dot{\varepsilon}_{x}=\dot{u}_{x}=+1$, la dissipation obtenue par régularisation, notée $d$ dans la section 4, s'exprimera en fonction du coefficient de Poisson fictif comme suit :

$$
d(v)=2 \frac{\sigma_{Y}}{\sqrt{3}}-\frac{\sigma_{Y}^{2}}{4 \mu}=2 \frac{\sigma_{Y}}{\sqrt{3}}-2 \sigma_{Y}^{2} \frac{(1-2 v)(1+v)}{E}
$$

pour laquelle la valeur du coefficient de Poisson fictif, $v=0.5$, coïncide exactement avec la valeur analytique, $2 k$. Nous notons que la dissipation régularisée coïncide avec la charge limite, en d'autres termes, le graphe de la figure 1 illustre la variation de la charge limite régularisée en fonction du coefficient de Poisson.

\subsection{Estimation de $E^{*}$ et $v^{*}:$ un exemple numérique}

Cet exemple se base sur une application largement utilisée pour la comparaison des approches, c'est le problème de poinçonnement en état plan de déformation ayant pour dimensions celles qui figurent sur le dessin, figure 2. Le maillage adopté est constitué de 98 éléments finis rectangulaires quadratiques. Le matériau est considéré rigide parfaitement plastique et son seuil de cisaillement est $k=\sigma_{Y} / \sqrt{3}$. Notre attention est focalisée sur les influences des deux grandeurs fictives de régularisation : le module de Young et le coefficient de Poisson. On a l'habitude de définir le facteur de poinçonnement suivant : $F_{p}=\frac{\text { Charge limite }}{k \mathrm{x} \text { section poincon }}$.

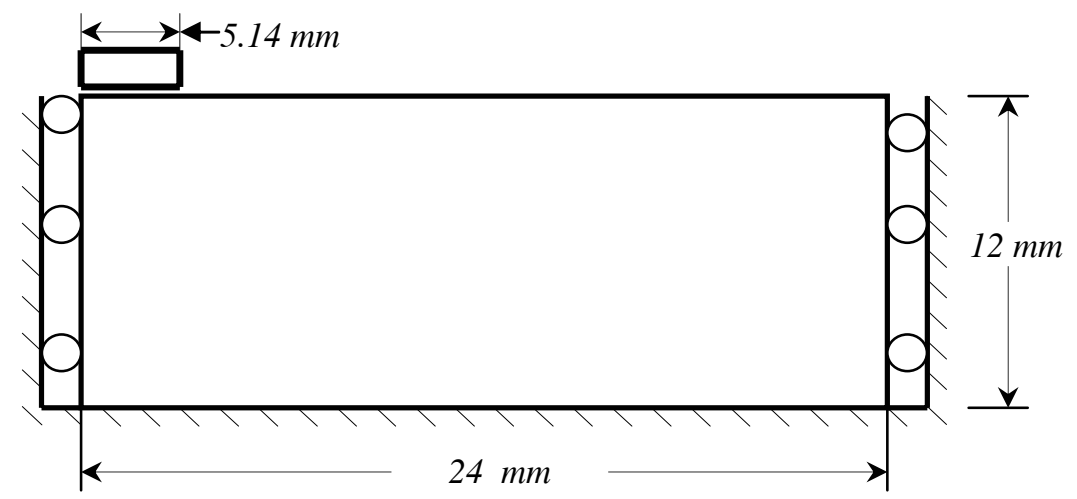

Figure 2. Modèle mathématique et conditions aux limites 


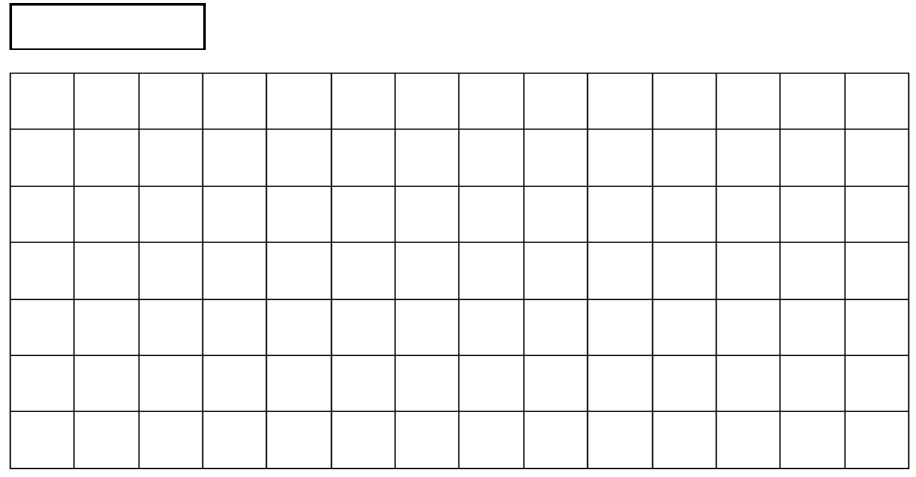

Figure 3. Modèle éléments finis : 98 éléments finis rectangulaires quadratiques

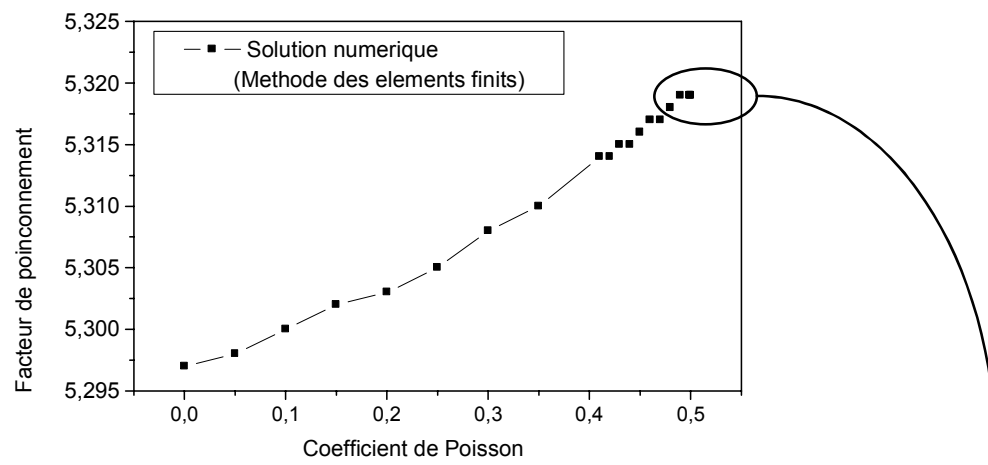

Figure 4. Facteur de poinçonnement en fonction du coefficient de Poisson fictif
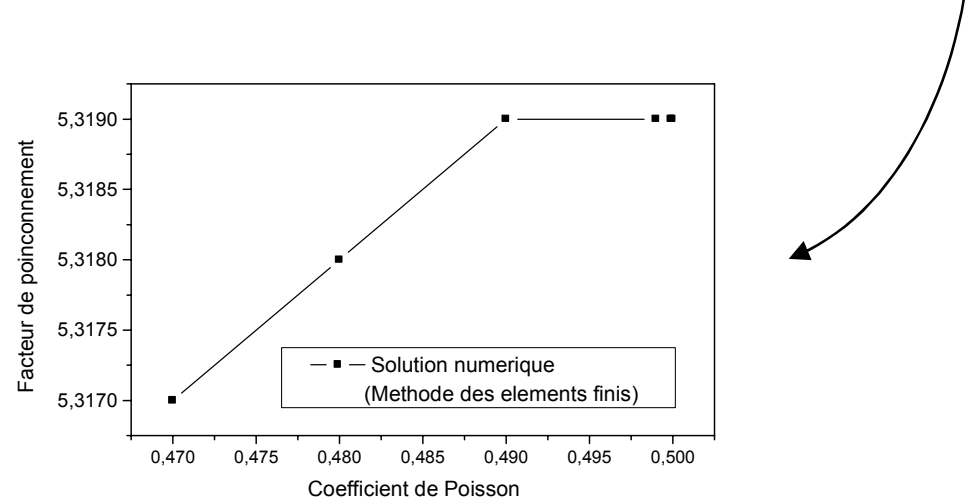

Figure 5. Facteur de poinçonnement en fonction du coefficient de Poisson fictif 


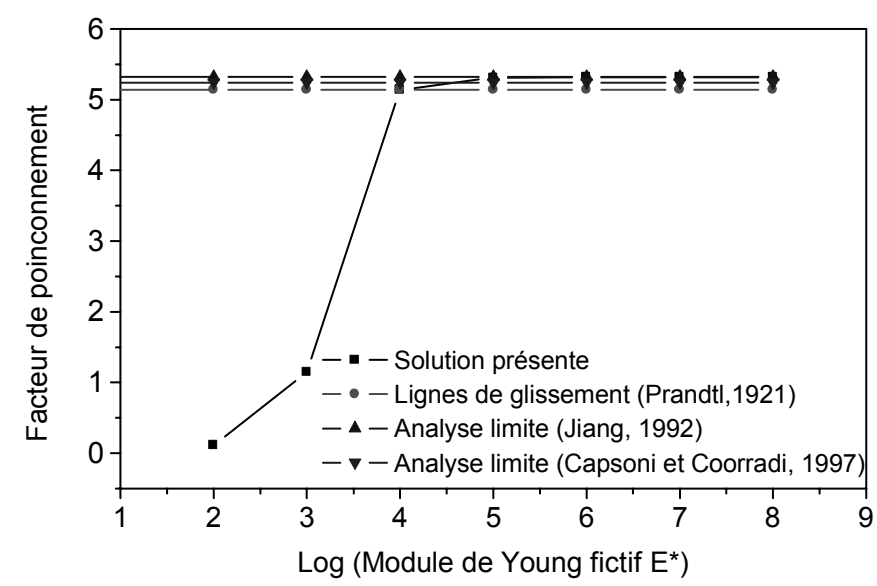

Figure 6. Facteur de poinçonnement en fonction du module de Young fictif

Sur les figures 4 et 5 , nous avons tracé le facteur de poinçonnement pour une plage de variation du coefficient de Poisson allant de 0 à 0,4999 . Malgré cette plage de variation importante de ce coefficient, la variation relative du facteur de poinçonnement n'est que de l'ordre de 0,40\%. Par ailleurs, la figure 6 montre l'évolution de ce facteur en fonction du module de Young fictif sur une plage de variation de $10^{2}$ à $10^{8}$, en comparaison avec les solutions des références (Prandtl, 1921, Jiang, 1992 et Capsoni et corradi, 1997). Il apparaît que pour des valeurs plus grandes de cette grandeur fictive, le calcul devient très lourd et ne fournit pas plus de précision. En se basant donc sur les résultats de la figure 6, il est simple de conclure qu'à partir de la valeur $10^{4}$ ou même $10^{5}$ le facteur de poinçonnement et donc la charge limite gardent une valeur presque constante. En conclusion, les valeurs numériques que nous retiendrons pour la suite sont $E^{*}=10^{5}$ et $v^{*}=0.49$.

\section{Evolution de la charge limite en présence de l'écrouissage}

La question de la notion de charge limite en présence de l'écrouissage est abordée au moyen de l'approche de l'analyse limite séquentielle, qui est une procédure basée sur la méthode cinématique (au sens de l'analyse limite). Cette approche est développée dans l'objectif de suivre l'évolution des grandes déformations en tenant compte de l'écrouissage isotrope. En effet, l'analyse limite séquentielle consiste à résoudre une séquence de problèmes d'analyse limite en actualisant, à la fin de chaque séquence, la géométrie et la contrainte limite. La géométrie est actualisée à travers les coordonnées des différents points du solide en multipliant le champ de vitesse par un incrément de temps suffisamment petit 
(hypothèse de petite perturbation). De même pour l'actualisation du seuil de plasticité, puisque l'écrouissage isotrope peut se traduire par l'évolution de ce seuil en fonction de la déformation plastique accumulée qu'on peut calculer en fonction du champ des déformations, c'est-à-dire en fonction du champ des vitesses.

Dans la suite, nous considérons ici un bloc de matériau en état plan de déformation, en compression entre deux plateaux rigides sans frottement. Les dimensions initiales du bloc sont $L x H$. En tenant compte de la double symétrie, on considère seulement le quart de la pièce. La structure est discrétisée en 144 éléments finis rectangulaires linéaires (4 nœuds et 8 degrés de liberté). Dans une première étape, le matériau constitutif est supposé rigide parfaitement plastique et nous sommes intéressés par l'influence de l'actualisation de la géométrie sur la charge limite; puis dans une seconde étape, l'objectif consiste à mettre en évidence l'influence de l'écrouissage isotrope, également sur la charge limite. Pour cela, notons par $h$ la distance initiale entre la tête de l'outil ( $0 \leq h \leq H)$ qui se déplace du haut vers le bas, l'outil pourra être monté par exemple sur la partie mobile d'une presse, permettant ainsi de contrôler les séquences de compression. On définit le facteur de charge $\alpha$ par le rapport entre la charge limite et le produit de la section par $2 k$. Les résultats fournis par notre programme sont tracés sur la figure 7 , en fonction de $(1-h / H)$ en les comparant avec la solution analytique disponible (Hwan, 1992) qui tient compte du changement de géométrie (pas d'écrouissage). Le champ des vitesses nodales est représenté pour les deux cas : $0 \%$ et $50 \%$ de réduction de la hauteur.

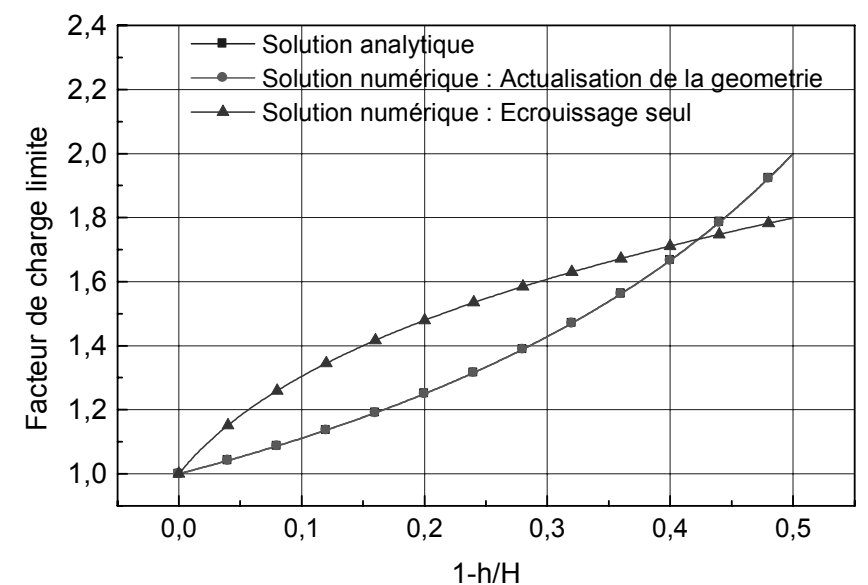

Figure 7. Facteur de charge limite en fonction de $(1-h / H)$

Nous pouvons noter le très bon accord de la solution numérique avec la solution analytique ; d'après le graphe (figure 7), les deux courbes (analytique et numérique), où seulement l'actualisation de la géométrie est prise en compte, sont presque 
superposées. Puisque le frottement est négligé entre l'outil et la pièce, la déformation est de type homogène et nous pouvons remarquer que les éléments ont gardé leur forme géométrique initiale sans distorsion bien qu'on ait atteint $50 \%$ de la hauteur. Il n'est pas donc nécessaire dans ce cas de réaliser un remaillage. Cependant, pour des applications plus complexes comme le cas du poinçonnement où les contraintes varient rapidement d'un point à un autre du milieu déformé, il sera nécessaire après un certain nombre de séquences de déformation, de réaliser un nouveau maillage (remaillage).

Dans la suite de l'application, nous étudierons le cas du matériau avec écrouissage isotrope. Nous retenons donc la notation précédente et considérons que le matériau a une contrainte limite d'écoulement plastique évoluant selon la loi d'écrouissage $\beta\left(\varepsilon_{p}\right)=\frac{\sigma_{Y}^{a}}{\sigma_{Y}}=\left(1+16.4 \varepsilon_{p}\right)^{0.25}$. Les résultats obtenus sont montrés sur les figures 8 et 9 , où le facteur de charge est représenté en fonction de $(1-h / H)$ en comparaison avec le cas sans écrouissage et aussi avec les résultats numériques de la référence (Hwan, 1992). Comme il est prévu, la charge limite du cas avec écrouissage est plus grande à celle correspondant au matériau sans écrouissage. Nous pouvons noter le très bon accord de la solution obtenue avec celle de la référence (Hwan, 1992) où l'auteur a adopté un algorithme itératif, dit d'approximations successives, pour la résolution du problème cinématique d'analyse limite; cet algorithme (Hwan, 1992) approxime le problème de minimisation sans contrainte par une série de problèmes de programmation quadratique et résout un problème de programmation quadratique dans chaque itération. La condition d'incompressibilité est traitée au moyen de la méthode de pénalisation, contrairement à l'approche que nous avons proposée où cette condition est abordée de manière systématique au moyen de l'inf-convolution.

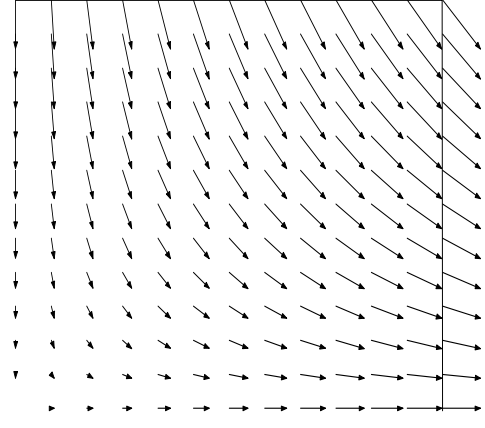

(a)

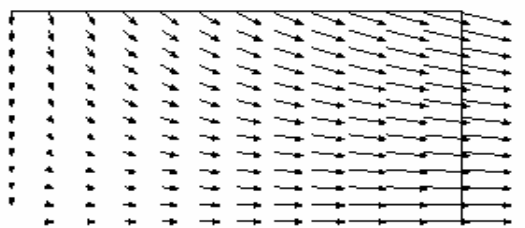

(b)

Figure 8. Champs des vitesses nodales : a) $0 \%$ et b) $50 \%$ 
Nous pouvons remarquer à nouveau pour ce problème relativement simple la superposition de la présente solution sur celle de (Hwan, 1992) où les effets de l'écrouissage et du changement de la géométrie sont considérés ensemble (figure 9). Cette figure met en évidence aussi la grandeur du facteur de charge limite dans les deux cas, avec ou sans écrouissage.

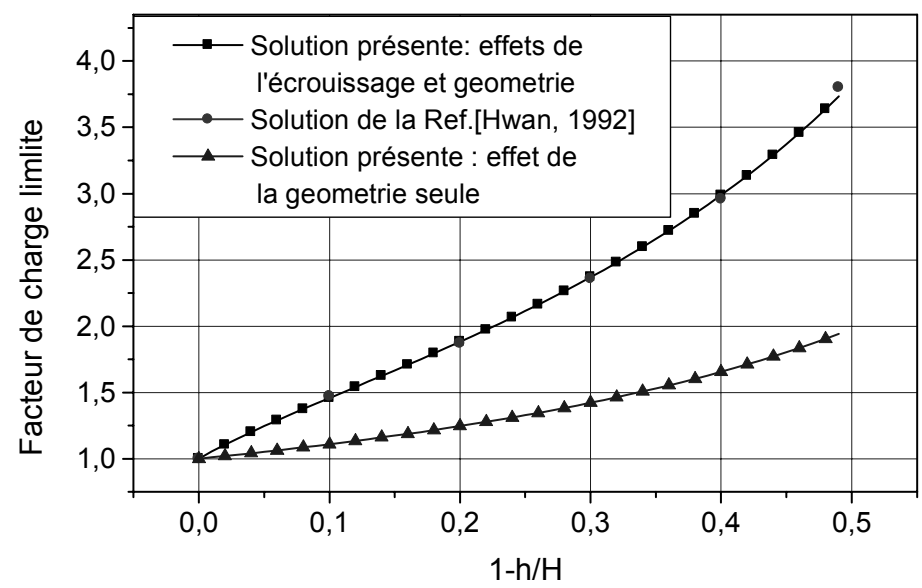

Figure 9. Charge limite en fonction de $(1-h / H)$

\section{Conclusion}

Nous avons présenté un algorithme pour le calcul de l'état limite des métaux par l'approche cinématique au sens de l'analyse limite en adoptant la méthode des éléments finis et en tenant compte de l'écrouissage isotrope et de l'aspect grandes déformations. Afin de surmonter la difficulté entraînée par la non-différentiabilité de la dissipation, nous avons adopté la méthode de régularisation par inf-convolution qui nous a permise de calculer facilement le gradient de la dissipation plastique et les contraintes aux points de Gauss. L'écrouissage et l'aspect grandes déformations sont introduits de manière séquentielle par actualisation du seuil de plasticité et de la géométrie après chaque séquence de résolution du problème d'analyse limite [14]. Le processus d'actualisation est basé sur la connaissance du champ de vitesse aux nœuds, obtenu après résolution de [14]. La séquence suivante d'analyse limite est conduite après avoir actualisé la géométrie en ajoutant les incréments de déplacement, obtenus par multiplication du champ de vitesse par un incrément de temps suffisamment petit, aux coordonnées des nœuds ; et de manière similaire, le seuil de plasticité est actualisé en mettant à jour la déformation plastique accumulée introduite dans la loi [2]. En se servant de cet algorithme, nous pouvons suivre l'écoulement plastique de la pièce en grande déformation s'approchant ainsi de la réalité. Dans ce travail, nous nous sommes intéressés seulement à la loi du matériau, cependant, la prise en compte du phénomène du contact avec frottement sur les interfaces ne manque pas d'intérêt. En effet, les 
processus de mise en forme qui pourraient être les domaines les plus intéressés par les résultats obtenus ne peuvent négliger, dans la majorité des situations, ce phénomène. Actuellement, on dispose de la formulation théorique de l'analyse limite tenant compte du contact et du frottement (De Saxcé et Bousshine, 1998) et d'une application relativement simple dans le cas d'analyse limite de structures de portique avec appuis frottants (Bousshine et al., 2002). La suite naturelle de ce travail impose donc d'aborder l'effet du contact et du frottement lors du processus de déformation par le biais de l'approche d'analyse limite.

\section{Bibliographie}

Avitzur B., Metal forming: Processes and Analysis, McGraw-Hill, New York, 1968.

Avitzur B., Handbook of Metal forming Processes, Wiley, New York, 1983.

Bousshine L., Chaaba A., de Saxcé G. et Guerlement G., «Etat limite des métaux rigides parfaitement plastique par l'analyse limite et la méthode des éléments finis », Les cahiers de la recherche, série A : Sciences et Techniques, vol. 4, Septembre, 2002, p. 145-160.

Bousshine L., Chaaba A. et de Saxcé G., "Plastic limit load of frictional contact supports plane frames", lnt. Jour. of Mech. Sci., November 2002, vol. 44, p.2189-2216.

Capsoni A. et Corradi L., "A finite element formulation of the rigid plastic limit analysis problem", Int. Jour. Num. Engng, vol. 40, p. 2063-2086.

Chaaba A., Bousshine L. et de Saxcé G., "Kinematic limit analysis modelling of rigid perfectly plastic material by a regularisation approach and finite element method", International Journal for Numerical Methods in Engineering, 2003, 57, p. 1899-1922.

Chaaba A. Analyse limite des métaux et des matériaux granulaires par la théorie du bipotentiel. Formulation éléments finis et applications, Thèse de Doctorat en Sciences Appliquées, ENSEM de Casablanca, Janvier, 2001.

Chen W.F. Limit analysis and Soil Plasticity, Elsevier, New York, 1975.

Corradi L., Panzeri N. "A triangular finite element for sequential limit analysis of shells", Advances in Engineering software, 35, 2004, p. 633-643.

De Saxcé G. et Bousshine L., "Limit Analysis Theorems for Implicit Standard Materials: Application to the unilateral Contact with Dry Friction and Non-associated flow Rules in Soils and Rocks", Int. Jour. Mech. Sci., 1998, vol. 40, n 4, p. 387-398.

Drucker D.C., Greenberg, H.J. et Prager W., "The safety factor of an elastic-plastic body in plane strain", J. Appl. Mech., 18, 1951, p. 371-378.

Drucker D.C., Prager W. et Greenberg, H.J., "Extended limit design theorems for constitutive media", Quart. Appl. Math., 9, 1952, p. 381-389.

Fassi-Fihri H., Bousshine L., Elharif A. et Chaaba A., « Analyse élastoplastique des métaux en présence du contact unilatéral avec frottement sec de Coulomb», Les cahiers de la recherche, série A : Sciences et Techniques, vol. 4, Septembre, 2004, p. 145-160. 
Fassi Fihri H., Bousshine L., Chaaba A. et Elharif A., "Numerical simulation of orthogonal metal cutting by incremental elastoplastic analysis and finite element method", Journal of Material Processing Technology, 141, 2003, p. 181-188.

Friaâ A., Le matériau de Norton-Hoff généralisé et ses applications en analyse limite, C.R. Acad. Sci. Paris, t. 286, Série A, pp. 953-956, 1978.

Friaâ A., La loi de Norton-Hoff généralisée en plasticité et viscoplasticité, Thèse de Doctorat d'État, Paris VI, 1979.

Gaudrat V.F. "A Newton type algorithm for plastic limit analysis", Computer Methods in Applied Mechanics and Engineering, vol. 88, n² 2, July 1991, p. 207-224.

Greenberg H.J. et Prager W., "Limit Design of beams and frames”, Proc. ASCE, vol. 77, $\mathrm{n}^{\circ} 59,1951$

Guennouni A. T., Matériau de Norton-Hoff pour divers critères de plasticité de mécanique des sols, Thèse de Doctorat, ENPC, Paris, 1982.

Guennouni A. T., Letallec P., «Calcul à la rupture: Régularisation de Norton-Hoff et lagrangien augmenté », J. Mé. Théorique et Appliquée, vol. 2, n 1, 1982.

Halphen B. et Salençon J., Elsatoplasticité, Presse de l'Ecole Nationale des Ponts et Chaussées, 1987.

Hill R., "On the state of stress in a plastic-rigid body at the yield point", Phil. Mag., vol. 7 , $\mathrm{n}^{\circ} 42,1951$, p. $868-875$.

Hill R., "On note on estimating yield-point loads in a plastic-rigid body", Phil. Mag. vol. 7, $\mathrm{n}^{\circ} 43,1952$, p. $353-355$.

Hill R., The mathematical Theory of Plasticity, Oxford Sciences publications, 1950.

Horne MR, Merchant W., The stability of frames, London, Maxwell, 1965.

Huh H., Kim K.P. et Kim H.S., "Collapse simulation of tubular structures using a finite element limit analysis approach and shell elements", Int Jour. of Mech. Sci., 2001, 43 p. 2171-2187.

Huh H., Lee C.H. et Yang W.H., "A general algorithm for plastic flow simulation by finite element analysis", lnt. Jour. Solids and Structures, 1999, 36, p. 1193-1207.

Hwan C. L., Large plastic deformation by sequential limit analysis: A finite element approach with application in metal forming, Ph.D. Thesis, University of Michigan, 1992.

Jiang G.L., Application de l'analyse limite à l'étude de la stabilité des massifs de sol, Thèse de Doctorat de 14ENPC, Septembre, 1992, Paris.

Johnson W., Sowerby R. et Venter R.D., Plane-Strain Slip-Line Fields For MetalDeformation Processes, Pergamon Press, 1982.

Jospin N.D., Etats limites des coudes par la méthode des éléments finis et la programmation mathématique, Thèse de Doctorat en Sciences Appliquées, Université de Liège. 1992.

Kim Y.J. et Yang D.H. “A formulation for rigid-plastic finite element method, considering work-hardening effect”, Int. J. Mech. Sci., 27 1985, p. 487-495. 
Kobayashi S., Oh S. I. et Altan T., Metal forming and the finite element method, Oxford Univ. Press, 1989.

Koiter W.T., "General Theorems for elastic-plastic solids", Progress in solid mechanics, vol. I, Sneddon \& Hill. ed., North Holland, 1960, p. 163-221.

Lee C. H. And Kobayashi S., "New solutions to rigid-plastic deformations problems using a matrix method", Transactions of the ASME, J. Eng. Ind., 95, 1973, p. 865-886.

Lemaitre J. et Chaboche J.L., Mechanics of solid materials, Cambridge University Press, Cambridge, 1990.

Leu S. Y., "Limit analysis of strain-hardening viscoplastic cylinders under internal pressure by using the velocity control: Analytical and numerical investigation", International journal of Mechanical Sciences, 50, 2008, p. 1578-1585.

Leu S. Y., "Analytical and numerical investigation of strain-hardening vicscoplastic thickwalled cylinders under internal pressure using sequential limit analysis", Compu. Methods Appl. Mech Engrg., 196, 2007, p. 2713-2722.

Lung M. et Marenholtz O., "A finite element procedure for analysis of metal forming process", Transactions of the CSME, 2, 1974, p. 31-36.

Mercier B., « Sur la théorie et l'analyse numérique de problèmes de plasticité », Thèse d'Etat, Université, Paris VI, 1977.

Moreau J. J., « Proximité et dualité dans un espace Hilbertien », Bull. Soc. Math., France, 93, 1965 , p. $273-299$

Mori K., Osakada K. et Oda T. "Simulation of plane strain rolling by the rigid-plastic finite element method", Int. J. Mech. Sci., 24, 1982, 519.

Murtagh RA., et Saunders M.A., Minos 5.1 user's guide, Stanford University, 1987.

Nagtegaal J.C., et De Jong J.E., "Some computational aspects of elastic-plastic large strain analysis", Int. J. Num. Meth. Eng., 17, 1981, p. 15-41.

Ponter A.R.S., "Carter K.F. Limit state solutions, based upon linear solutions with a spatially varying elastic modulus", Meth. Appl. Mech. Eng., 140, 1997, p. 237-258.

Ponter R.S., Fuschi P. et Engelhart M., "Limit analysis for a general class of yield conditions", Eur J Mech A/Solids, 2000, 19, p. 401-21.

Prager W., "The general theory of limit design", Proc., $8^{\text {th }}$ Int. Congress Appl. Mech., vol. 2, Istambul, 1952, p. 65-72.

Prandtl L., "On the penetration hardness of plastic materials and the hardness of indenters", Zeits. Ang. Math. Mech., vol., 1921, p. 15.

Rockafellar R.T., Convex Analysis, Princeton University Press, 1996.

Salençon J., Calcul à la Rupture et Analyse Limite, Presses de l'ENPC, 1983.

Save M. et Massonet Ch., «Calcul plastique des constructions », vol. II, Structures Spatiales, Structures dependant de plusieurs parameters, $2^{\mathrm{e}}$ éd., CBLIA, Bruxelles, 1972. 
Voldoire F., "Regularized limit analysis and applications to the load carrying capacities of mechanical components", ECCOMAS, 2000, 11-14 September 2000, Barcelona, Spain.

Voldoire F., Limit analysis by the Norton-Hoff-Friaâ regularising method, M. Heitzer, M. Staat, LISA project report 2001, publication du John von Neumann Institute for Computing, 2003.

Wagoner R.H., Chenot J.L., Metal Forming Analysis, Cambridge University Press, 2001.

Yang W. H., "Large deformation of structures by sequential limit analysis", Int. Jour. Solids and Struc., 1993.

Yoon J.H. et Yang D.H., "Rigid-plastic finite element analysis of three dimensional forging by considering friction on continuous curved dies with initial guess generation”, Int. J. Mech. Sci., 30, 1988, p. 887-898.

Received: 2 December 2008

Accepted: 14 May 2009 
